\title{
Personalized genome assembly for accurate cancer somatic mutation discovery using cancer-normal paired reference samples
}

Chunlin Xiao ${ }^{1 *}$, Zhong Chen ${ }^{2}$, Wanqiu Chen², Cory Padilla ${ }^{3}$, Li-Tai Fang ${ }^{4}$, Tiantian Liu², Valerie Schneider ${ }^{1}$, Charles Wang ${ }^{2}$, and Wenming Xiao ${ }^{5^{*}}$

${ }^{1}$ National Center for Biotechnology Information, National Library of Medicine, National Institutes of Health, 45 Center Drive, Bethesda, MD 20894;

${ }^{2}$ Center for Genomics, Loma Linda University School of Medicine, 11021 Campus St., Loma Linda, CA 92350;

${ }^{3}$ Dovetail Genomics, 100 Enterprise Way, Scotts Valley, CA 95066;

${ }^{4}$ Bioinformatics Research \& Early Development, Roche Sequencing Solutions Inc., 1301 Shoreway Road, Belmont, CA 94002;

${ }^{5}$ The Center for Drug Evaluation and Research, U.S. Food and Drug Administration, Silver Spring, MD, USA

*Correspondence should be addressed to: xiao2@mail.nih.gov and Wenming.Xiao@fda.hhs.gov

\section{Abstract}

The use of personalized genome assembly as a reference for detecting the full spectrum of somatic events from cancers has long been advocated but never been systematically investigated. Here we address the critical need of assessing the accuracy of somatic mutation detection using personalized genome assembly versus the standard human reference assembly (i.e. GRCh38). We first obtained massive whole genome sequencing data using multiple sequencing technologies, and then performed de novo assembly of the first tumor-normal paired genomes, both nuclear and mitochondrial, derived from the same donor with triple negative breast cancer. Compared to standard human reference assembly, the haplotype phased chromosomal-scale personalized genome was best demonstrated with individual specific haplotypes for some complex regions and medical relevant genes. We then used this well-assembled personalized genome as a reference for read mapping and somatic variant 
discovery. We showed that the personalized genome assembly results in better alignments of sequencing reads and more accurate somatic mutation calls. Direct comparison of mitochondrial genomes led to discovery of unreported nonsynonymous somatic mutations. Our findings provided a unique resource and proved the necessity of personalized genome assembly as a reference in improving somatic mutation detection at personal genome level not only for breast cancer reference samples, but also potentially for other cancers.

\section{Introduction}

Accurately detecting somatic mutations and subsequently understanding genomic instability in cancer are critical for precision cancer therapies, and many genomics studies, including tremendous efforts from the renowned TCGA and ICGC consortia, have been directed to investigate genomic instability of cancer, and greatly improved our understanding of cancer biology [1-4]. Most recently, tumor-normal pair reference samples and reference callsets were established by the SEQC-II consortium for benchmarking somatic mutation detections from different sequencing platforms and bioinformatic analysis methods [46-47]. This standard is an indispensable foundation and resource for assessing the accuracy and reproducibility of somatic mutation profiling in cancer cell lines.

To date, discovering cancer somatic events and defining high-confidence reference somatic callsets mainly rely on a standard human reference assembly (such as GRCh38) as a benchmark for sequencing analysis. However, the GRCh38 has its own limitations. Although of very high quality, it remains incomplete due to some unresolved assembly issues and persistent gaps, including those at centromeres, telomeres, and heterochromatic regions [5-8]. Incorrect or missing sequences in GRCh38 reference assembly may lead to failed or spurious read mapping and unreliable subsequent analysis results (namely reference bias [9]). Moreover, the human reference assembly was derived from the DNAs sourced from multiple individuals, though approximately $70 \%$ of the GRCh38 sequences were contributed by a single Africa-European admixed male (RP11) [6]. Such mosaic haplotype representation in the reference assembly may 
complicate the identification of somatic variations from cancer samples; therefore, use of a de novo assembly of the personalized genome, rather than the standard reference assembly, for confident cancer mutation discovery has been advocated $[5,7,10]$. There is more to be learned from direct comparison of the tumor genome to the normal genome from which it is derived, than to an unrelated, random, mosaic genome like the latest GRCh38 reference genome.

The advancements of DNA sequencing technologies provide an extraordinary opportunity to perform de novo assembly for individual genomes at affordable cost. Particularly, the breakthrough of long range DNA sequencing from the third-generation sequencing technologies now makes it possible to accurately assemble individual genomes to near completion, which has been done for several samples, including HX1 [11], AK1 [12], NA12878 [13], CHM13 [14], and HG002 [15]. These studies demonstrated the recent advancements in genome assembly methods and subsequent germline variant detection. However, using personalized genome as reference for somatic mutation detection in cancer, particularly in cancer-normal paired samples, has not been systematically investigated. Here we present our work that combined multiple sequencing technologies, including Illumina short reads, 10X Genomics linked reads, PacBio long reads, and Hi-C (high-throughput chromosome conformation capture) reads, to reconstruct, to our knowledge, the first tumor-normal paired genomes [7]. Derived from the same individual, HCC1395 cancer cell line, and a matched B lymphocyte cell line HCC1395BL [16] as well-studied reference samples by FDA-led SEQC-II consortium [46-47], represent one of the most important tumor-normal models for triple negative breast cancers (TNBC). This work will enable us to comprehensively assess the performance of somatic mutation detection with respect to the underlined reference assemblies being used, as well as pinpoint the complete spectrum of genomic alterations, using a personal genome as the reference.

\section{Results}

\section{Overall study design and establishing a reference-grade personal genome assembly}


We used five platforms for sequencing the normal reference sample (HCC1395BL B Lymphocyte cell line) and three platforms for sequencing the tumor reference sample (HCC1395 breast cancer cell line) from the same donor (Figure 1, Top). Using data from multiple sequencing technologies, including short reads, linked reads, and long reads (Supplementary Table 1), we built a workflow for establishing a de novo assembled genome (Figure 1, Bottom-Left). First, we generated initial assemblies for the HCC1395BL, using canu [17] for PacBio long reads and using Supernova [18] for 10X Genomics linked reads, respectively. Supernova assemblies contained many smaller contigs (size less than $10 \mathrm{~kb}$ ) than PacBio canu assemblies (Supplementary Table 2). Although the N50s and the largest scaffold of the Supernova assembly were much higher than the PacBio contig assembly (Supplementary Figure 1, Supplementary Figure 2), the latter was more complete as measured via Benchmarking Universal Single-Copy Orthologue (BUSCO) genes (Supplementary Figure 3). Additionally, a greater number of complete RefSeq proteincoding genes mapped to the PacBio assembly, and more base pairs from this assembly could be mapped to the GRCh38 reference (Supplementary Table 2). Taken together, these indicated that the overall quality of PacBio canu assembly was higher than Supernova assembly, particularly when gene content and the completeness are the primary concerns.

We then selected the PacBio contig assembly of HCC1395BL for further scaffolding. In general, two steps of scaffolding, first using $10 \mathrm{X}$ Genomics linked reads with ARCS, followed by Hi-C reads with SALSA (PacBio_canu + ARCS + SALSA), produced a better scaffolded assembly than using one-step scaffolding only (either PacBio_canu + ARCS or PacBio_canu + SALSA). The final scaffold assembly (hereafter referred to as HCC1395BL_v1.0) was the one with the highest Top50 (see "Methods") and scaffold N50 values, the largest scaffold size, and the greatest numbers of mapped complete BUSCOs and RefSeq transcripts (Table 1). HCC1395BL_v1.0 consisted of 1,645 scaffolds totaling $2.9 \mathrm{~Gb}$, of which $2.69 \mathrm{~Gb}(92.75 \%)$ is from Top50 scaffolds, with a scaffold N50 size of $69.97 \mathrm{Mb}$, in comparison to scaffold N50s 67.79 Mb for GRCh38 [6], and 44.84 Mb for AK1, a recent assembly from a diploid sample [12], respectively. 
had fewer scaffolds (1,645 vs. 2,832), a smaller L50 (14 vs. 21), and had much greater Top50 (2.69 Gb vs. $2.26 \mathrm{~Gb}), \mathrm{N} 50$ (69.97 Mb vs. $44.84 \mathrm{Mb})$, and largest scaffold size (181.21Mb vs. 113.92 Mb). Moreover, the HCC1395BL_v1.0 assembly contained more complete RefSeq NM (protein-coding) transcripts $(49,613$ vs. 49,432$)$ and RefSeq NR (non-protein-coding) transcripts $(15,227$ vs. 15,089$)$.

Consistency analysis (see "Methods") with the GRCh38 primary assembly (alt_loci excluded) showed that five chromosomes (chr4, chr8, chr14, chr18, and chr20) were near-completely covered by single scaffolds. The largest HCC1395BL_v1.0 scaffold (Scaffold_1 181.21Mb) covered more than $95 \%$ of GRCh38 chromosome 4. Four chromosomes (chr2, chr3, chr12, and chr19) were just broken at centromere regions. Several other chromosomal arms (chr1p, chr5p, chr6q, chr9p, chr10p, chr21q, and chrXq) were also covered by single HCC1395BL_v1.0 scaffolds (Figure 2).

Phasing analysis showed that 3.13 out of 3.17 million heterozygous sites were considered phased, and 6,368 phased blocks accounted for 2.42 Gb of HCC1395BL_v1.0, with the longest phased block 6.37 Mb (Supplementary Table 3). Approximately 15-fold coverage of Nanopore long reads were used to further extend phasing to $2.54 \mathrm{~Gb}$. The total number of phased blocks was subsequently decreased to 3,204 from 6,368 blocks, and the longest phased block was greatly improved, increasing from 6.37 Mb to $20.45 \mathrm{Mb}$ (Supplementary Table 3). With the phased assemblies (haplotype1 and haplotype2) for HCC1395BL cell line, we were able to call $4,115,622$ germline SNVs in diploid regions of autosomal chromosomes.

As a comparison, we also generated a de novo assembly for the HCC1395 cancer cell line, using both canu for PacBio long reads and Supernova for 10X Genomics' linked reads. The resulting HCC1395 assembly was more fragmented than HCC1395BL assembly (Supplementary Table 2), mainly due to high level of chromosomal aneuploidy and structural variations in this cancer cell line [19]. 


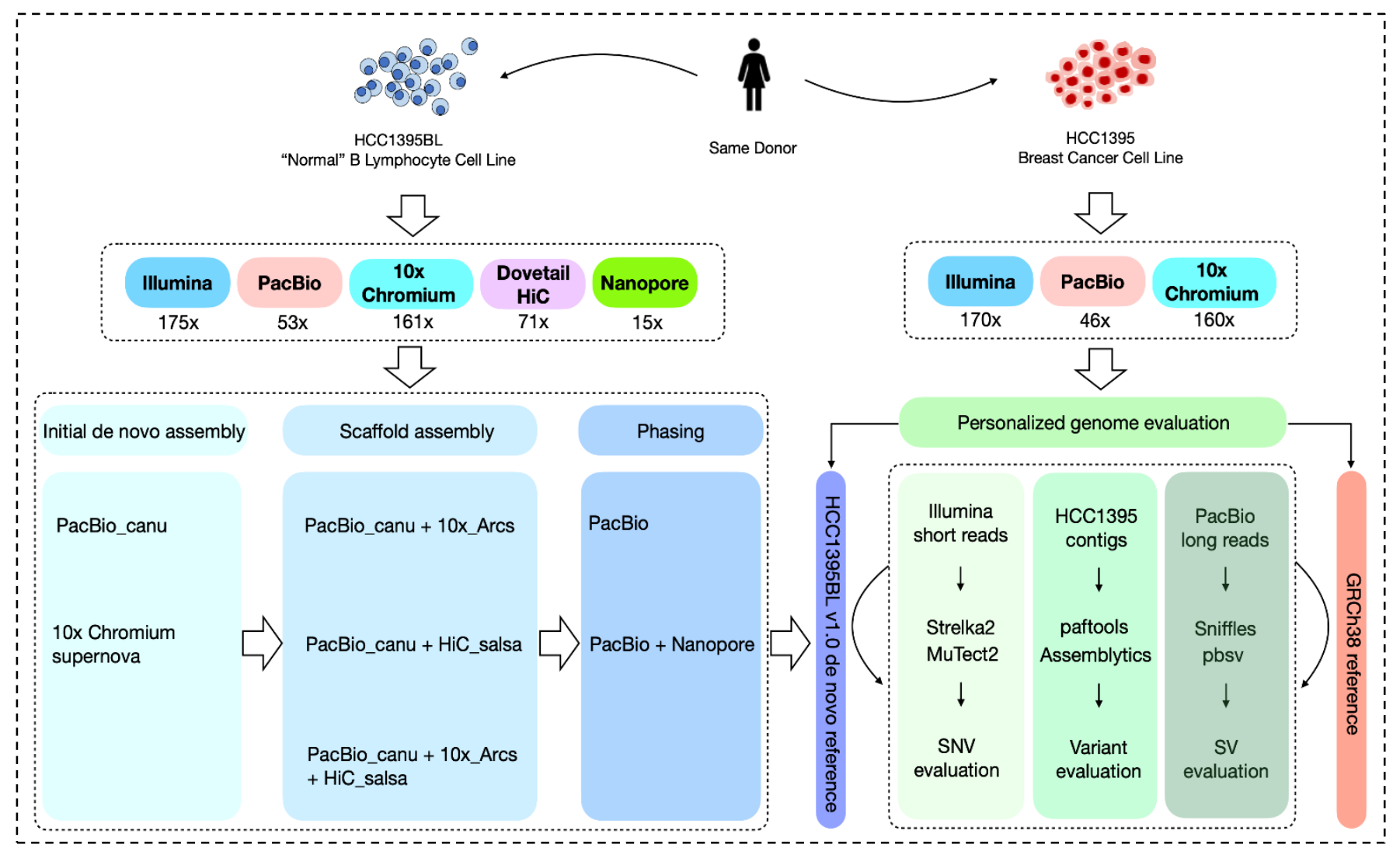

Figure 1. Schematic diagram of study design, DNA sequencing of the tumor-normal paired reference cell lines (HCC1395BL and HCC1395), de novo genome assembly of the cell lines, and assessment of somatic mutation detection using standard GRCh38 genome and personal genome assembly as references, respectively. 


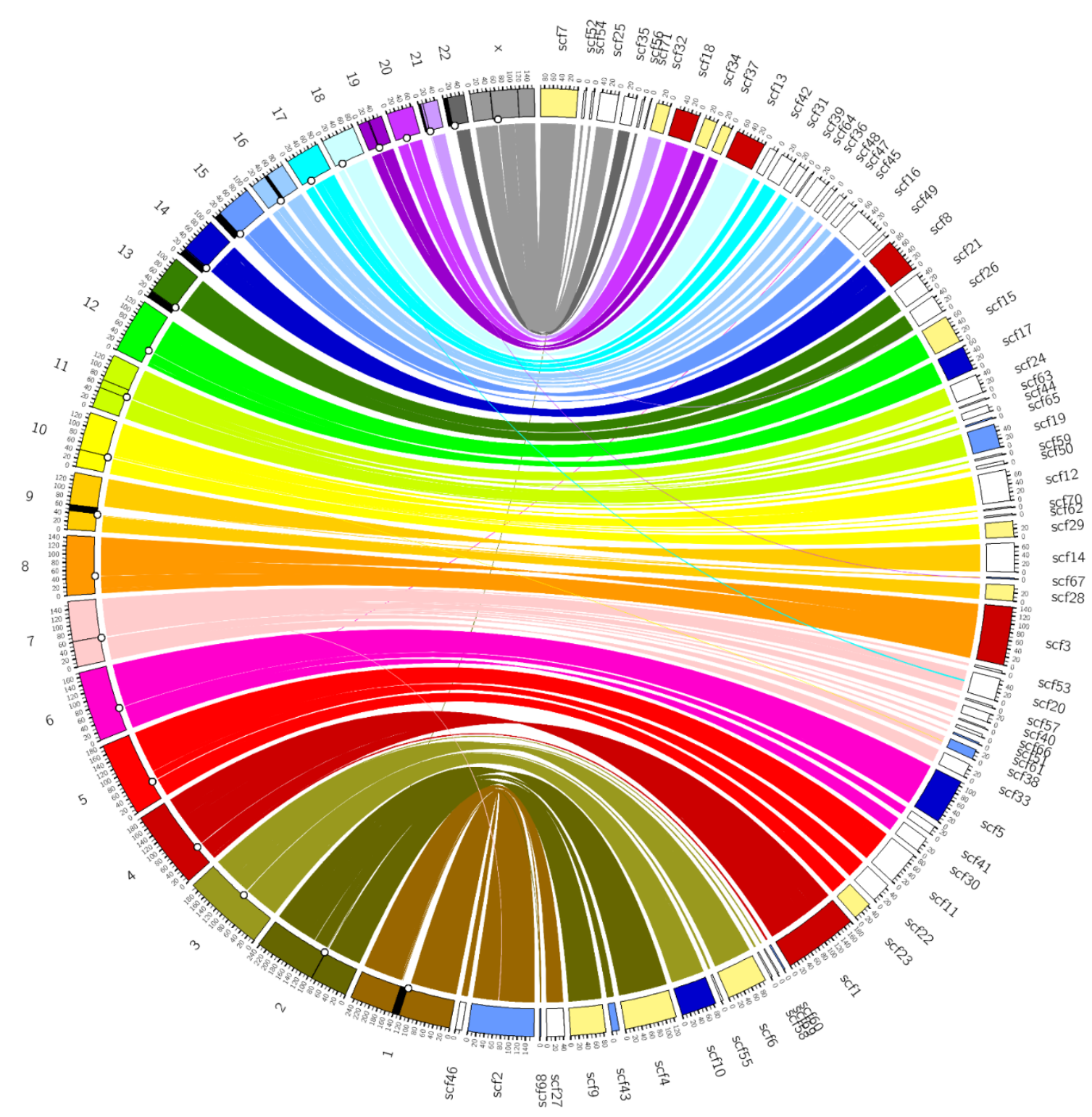

Figure 2. A Circos consistency plot of HCC1395BL_v1.0 (right side) against GRCh38 reference genome (left side). Included were 71 largest scaffolds with 2+ Mbp that accounted for $2,775,074,314$ bps (95.51\%). Shown here were alignments with coverage of $100 \mathrm{~Kb}+$ and mapping quality 60 on GRCh38 using minimap2. Centromeres were marked with black circle on the inner circle of GRCh38 chromosomes. Black regions on the chromosomes were GRCh38 gaps with $100 \mathrm{~Kb}+$ in size. Five chromosomes were near-completely covered by single scaffolds (Scaffold_1 for chr4, Scaffold_3 for chr8, Scaffold_8for chr14, Scaffold_13 for chr18, and Scaffold_18 for chr20, were colored with red color). Four chromosomes (chr2, chr3, chr12, and chr19) were broken only at centromere regions (near-completely covered by just two scaffolds). Centromere-crossing scaffolds were colored with light blue. Scaffolds (Scaffold_5, Scaffold_10, and Scaffold_17) covering one arm and crossing the centromere were colored dark blue. Scaffolds with near full coverage of one arm were covered with yellow. 
Table 1. Summary of quality assessments of assemblies from different scaffolding strategies.

\begin{tabular}{|c|c|c|c|c|c|c|c|c|c|c|c|c|}
\hline & $\begin{array}{c}\# \\
\text { scaffolds }\end{array}$ & $\begin{array}{l}\text { \# bps from } \\
\text { scaffolds }\end{array}$ & Top50 & N50 & L50 & $\begin{array}{c}\text { Largest } \\
\text { scaffold } \\
\text { length (bps) }\end{array}$ & $\begin{array}{c}\# \\
\text { scaffolds } \\
\text { on } \\
\text { GRCh38 }\end{array}$ & $\begin{array}{l}\text { \# novel } \\
\text { scaffolds }\end{array}$ & $\begin{array}{l}\text { \# bps from } \\
\text { novel } \\
\text { scaffolds }\end{array}$ & $\begin{array}{c}\# \\
\text { Complete } \\
\text { BUSCO } \\
\text { (4,104 } \\
\text { BUSCO) }\end{array}$ & $\begin{array}{c}\text { \# NMs } \\
95+\% \\
\text { mapped } \\
(50,052 \\
\text { NMs) }\end{array}$ & $\begin{array}{c}\text { \# NRs } \\
95+\% \\
\text { I mapped } \\
(15,544 \\
\text { NRs) } \\
\end{array}$ \\
\hline $\begin{array}{l}\text { PB_canu } \\
\text { (contigs) }\end{array}$ & 2,828 & $2,904,842,414^{1}$ & $\begin{array}{c}1,356,447,278 \\
(46.69 \%)\end{array}$ & $13,480,407$ & 757 & $62,208,403$ & 2,526 & 302 & $16,403,702$ & 3,890 & 49,287 & 15,115 \\
\hline $\begin{array}{c}\text { PB_canu + } \\
10 X_{\text {_arcs }} \\
\text { (scaffolds) }\end{array}$ & 2,032 & $2,904,931,213^{2}$ & $\begin{array}{c}2,067,627,443 \\
(71.17 \%)\end{array}$ & $35,058,531$ & 126 & $121,623,092$ & 1,764 & 268 & $14,867,391$ & 3,800 & 49,570 & 15,207 \\
\hline $\begin{array}{l}\text { PB_canu + } \\
\text { HiC_salsa } \\
\text { (scaffolds) }\end{array}$ & 1,891 & $2,905,381,691^{2}$ & $\begin{array}{c}2,377,981,926 \\
(81.84 \%)\end{array}$ & $46,871,224$ & 419 & $180,772,639$ & 1,617 & 274 & $15,303,825$ & 3,892 & 49,495 & 15,177 \\
\hline $\begin{array}{c}\text { PB_canu + } \\
\text { 10X_arcs + } \\
\text { HiC_salsa } \\
\text { (scaffolds, } \\
\text { HCC1395BL_v1.0) }\end{array}$ & 1,645 & $2,905,196,510^{2}$ & $\begin{array}{c}2,691,295,119 \\
(92.62 \%)\end{array}$ & $69,970,292$ & 214 & $181,209,810$ & 1,406 & 244 & $14,104,388$ & 3,889 & 49,613 & 15,227 \\
\hline
\end{tabular}

\section{Personal genome assembly includes individual specific haplotypes for clinically relevant} genes, and eliminates special handling of alt_loci/patches

We aligned a NCBI RefSeq transcript set (excluding all pseudogenes and genes from chromosome Y) to HCC1395BL_v1.0. 19,303 of 19,325 (99.89\%) RefSeq protein-coding genes could be successfully mapped onto HCC1395BL_v1.0 with minimum 95\% alignment identity and $50 \%$ alignment coverage, while 19,164 of 19,303 (99.27\%) these genes aligned at least $95 \%$ coverage (Figure 3A and Supplementary Table 4). Among RefSeq non-protein-coding genes, 10,049 of 10,061 (99.88\%) genes could be aligned to HCC1395BL_v1.0 successfully with minimum $95 \%$ identity and $50 \%$ coverage, with 9,958 of 10,049 (99.09\%) those genes covered at more than $95 \%$ in length (Figure 3A and Supplementary Table 4).

We next compared HLA gene family coverage in GRCh38 and HCC1395BL_v1.0, as an individual could have a very different HLA haplotype from another individual or the standard reference genome like GRCh38. HLA genes are located on the $6 p$ region of chromosome 6 , and previous cytogenetic analysis showed that this region from the HCC1395BL cell line was essentially 
haploid [46-47]. From the RefSeq gene set, 19 HLA genes (25 coding transcripts) are annotated on chromosome 6 of the GRCh38 primary assembly. We successfully identified all the HLA genes and corresponding transcripts in HCC1395BL_v1.0 and found that they are located on a single scaffold (Scaffold_30) aligning at minimum identity $95 \%$ and the minimum alignment coverage $95 \%$. Notably, the order of the HLA genes on this scaffold is identical to that on GRCh38 (Figure 3B). The only difference is the haplotype of HLA-DRB genes between HLA-DRA and HLA-DQA1, as the region of HLA-DRB genes is extremely divergent [20]. The haplotype of HLA-DRB in GRCh38 primary assembly was represented by HLA-DRB1 and HLA-DRB5 genes (human HLA-DR51 haplotype group [21]), but the HLA-DRB haplotype in HCC1395BL_v1.0 consists of HLA-DRB1 and HLA-DRB4 genes (human HLA-DR53 haplotype group), which is similar to the HLA-DRB haplotypes represented on GRCh38.p13 ALT_REF_LOCI_4 (NT_167246.2) and GRCh38.p13 ALT_REF_LOCI_7 (NT_167249.2). This demonstrated that the de novo assembly and scaffolding of HCC1395BL_v1.0 performed well on such hypervariable/complex regions that harbors HLA genes.

We also evaluated other clinically relevant genes whose only representations in GRCh38 are on alternate locus scaffolds, included in the reference to capture population diversity. HCC1395BL_v1.0 included GSTT1 (Glutathione S-transferase theta 1), and KIR2DL5A (Killer cell immunoglobulin like receptor, two Ig domains and long cytoplasmic tail 5A), two genes that are not included in the haplotypes represented on the chromosomes of the GRCh38 primary assembly. GSTT1, a gene previously localized to chromosome 22 of GRCh37 primary assembly, is found on alternate locus scaffold (NT_187633.1) in GRCh38. Likewise, for KIR2DL5 gene, the haplotype represented on Chromosome 19 with un-localized genomic contig (NT_113949.1) on the GRCh37 primary assembly included KIR2DL5A, but in the GRCh38 assembly, it is only found in alt_loci and novel patches (NT_113949.2). The changes in representations of these genes from GRCh37 to GRCh38 (or the future version) present analysis challenges when switching between versions of the standard reference genome. Additionally, because these genes are represented only on alt_loci and patches in GRCh38, and most existing tool chains do not handle those alternate locus scaffolds, they are consequently more difficult to study, their 
exclusion from analysis presents a heightened risk for misinterpretation of results. In contrast, as the individual specific haplotypes for these clinically relevant genes were correctly represented in the personalized assembly, no special handling of alt_loci or patches would be needed to assess them if HCC1395BL_v1.0 is used as reference as opposed to GRCh38.

A.

\section{RefSeq genes/transcripts mapping}

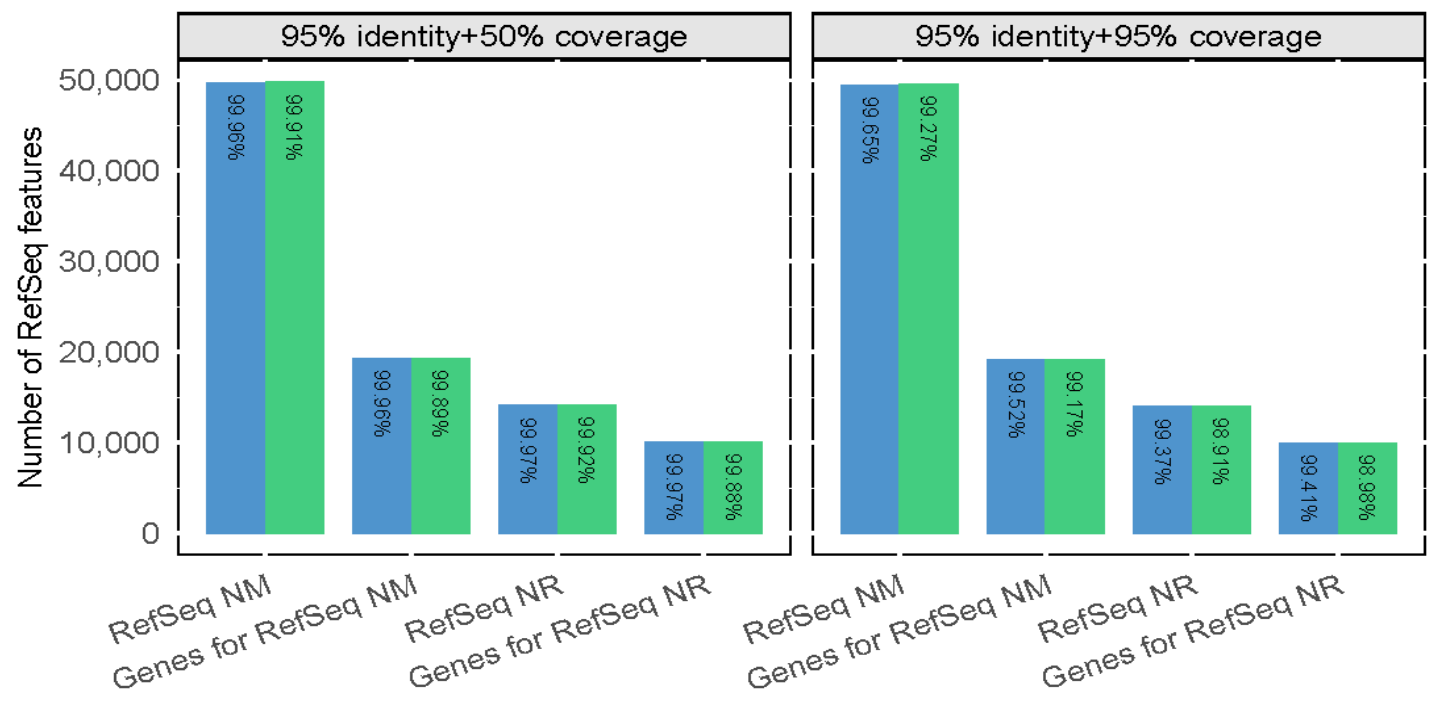

GRCh38 HCC1395BL_v1.0

$\begin{array}{rcccc} & \text { RefSeq NM } & \text { Genes for RefSeq NM } & \text { RefSeq NR } & \text { Genes for Refseq NR } \\ \text { Input } & 49,844 & 19,325 & 14,132 & 10,061 \\ \text { Found } & 49,838 & 19,322 & 14,131 & 10,060 \\ \text { Missing } & 6 & 3 & 1 & 1\end{array}$


B.

HLA genes on GRCh38 chr6
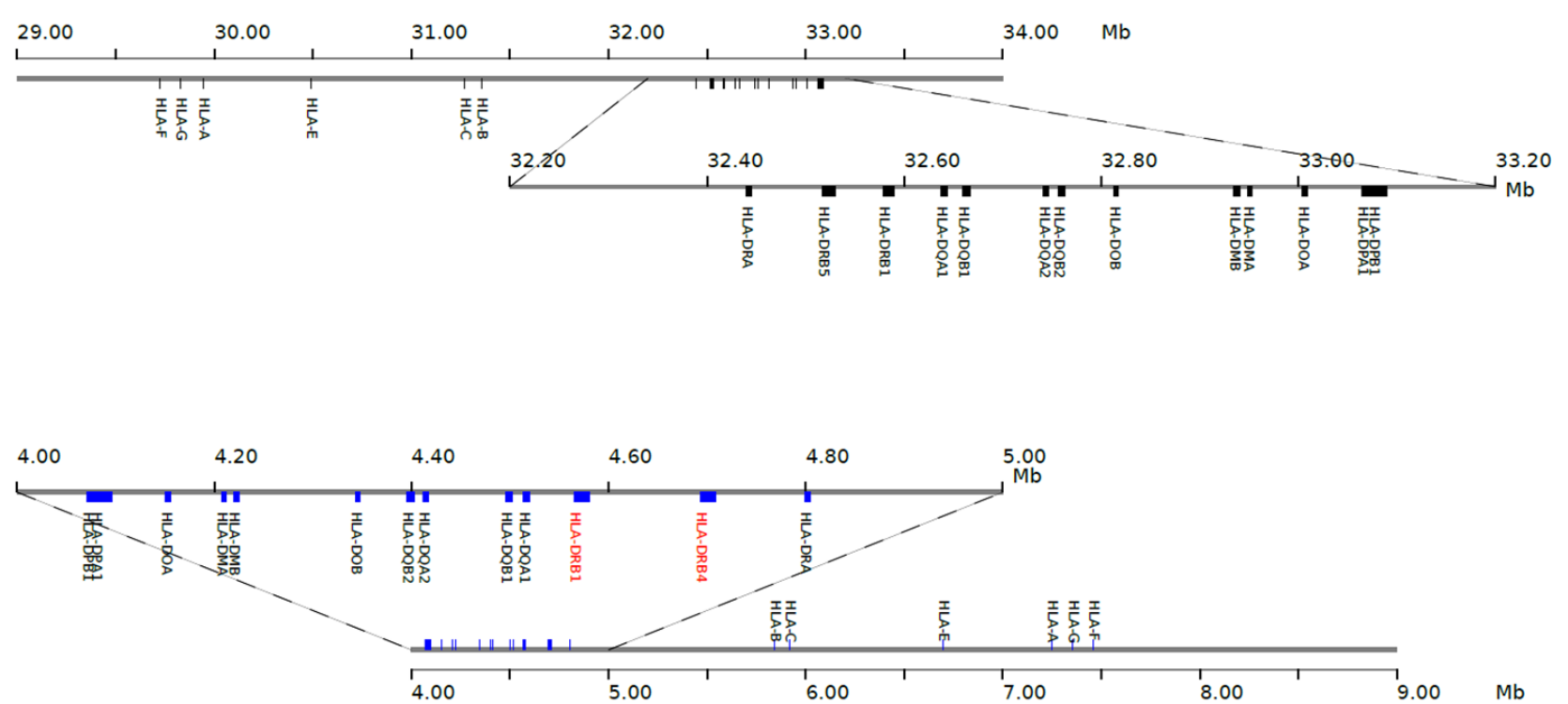

HLA genes on HCC1395BL Scaffold_30

Figure 3. (A) The summary of RefSeq genes/transcripts mapping on HCC1395BL_v1.0 and GRCh38; (B) HLA genes on Scaffold_30 of HCC1395BL_v1.0 in comparison to those on chromosome 6 of GRCh38 primary assembly. The haplotype of HLA-DRB (labels in red) in HCC1395BL_v1.0 consists of HLA-DRB1 and HLA-DRB4 genes (human HLA-DR53 haplotype group), while GRC38 primary assembly contains HLA-DRB1 and HLA-DRB5 genes (human HLADR51 haplotype group). The human HLA-DR53 haplotype was represented only in GRCh38 ALT_REF_LOCl sequences. Scaffold_30 was reverse complement mapped on GRCh38, but the order of HLA genes on chromosome or scaffold was identical between GRCh38 and HCC1395BL_v1.0.

\section{Personal genome assembly as reference enables better read mapping and accurate cancer somatic mutation detection}

We then used HCC1395BL_v1.0 as a reference for Illumina short read mapping and somatic variant discovery (Figure 1, Bottom-Right). While the mapping rates of short reads to the GRCh38 primary assembly (alt_loci excluded) and HCC1395BL_v1.0 were similar, we observed 
overall improved read mapping on HCC1395BL_v1.0 as opposed to GRCh38 (Figure 4A and Supplementary Table 5). For instance, a slightly higher percentages of properly-paired reads being mapped for both normal and tumor samples were seen on HCC1395BL_v1.0. Notably, the numbers of the improperly paired reads were reduced by $33 \sim 36 \%$, whereas the numbers of the mismatches for the mapped reads were decreased by about $16 \%$. In addition, the numbers of read alignments with soft-clipping were down by $12 \sim 13 \%$, while the numbers of read alignments with hard-clipping were down by 28 31\% (Supplementary Table 5).

Based on a previous study [22] and recent SEQC2 reports [46-47], two commonly used somatic mutation callers, namely Strelka2 [23] and MuTect2 [24], were selected for generating reports of somatic SNVs and small indels with the same settings based on same set of Illumina shortread data using HCC1395BL_v1.0 and GRCh38 (alt_loci excluded) as reference genomes, respectively. A higher number of overlapping calls between Strelka2 and MuTect2 were observed, and 1,983 more somatic SNVs/indels were seen on HCC1395BL_v1.0 than GRCh38 (Figure 4B and Supplementary Table 6).

For those 41,669 GRCh38-based somatic SNVs supported by both Strelka2 and MuTect2 callers (Supplementary Table 6), 40,768 SNVs (97.83\%) were successfully mapped on HCC1395BL_v1.0 with overlapping SNVs called by Strelka2/MuTect2 (Figure 4C and Supplementary Table 7). 682 SNVs (1.64\%) were able to map on HCC1395BL_v1.0 but without overlapping Strelka2/MuTect2 calls. Variant function analysis using ANNOVAR [25] showed that 120 of 682 SNVs were located in exonic or intronic regions, therefore including or excluding these sites would have an impact on downstream mutation interpretations (Supplementary Table 7). 219 SNVs (0.53\%) were considered as "not-mapped" on HCC1395BL_v1.0 due to the stringent mapping criteria (see "Methods"). Moreover, 3,995 SNVs (9.58\%) were considered as equivalent SNVs between GRCh38 and HCC1395BL_v1.0 but with mismatches in their flanking sequences (Supplementary Figure 4 and Supplementary Table 7). For example, the same set of reads was found (with mapping quality 60) to align across the corresponding intergenic SNV regions on HCC1395BL_v1.0 (scaffold_2:131886469-131886569 for SNV scaffold_2:131886519), but on 
GRCh38 (chr1:177753949-177754049 for SNV chr1:177753999) two mismatches (or two potentially homozygous germline SNVs) are observed in flanking sequences (Supplementary Figure 4A/4B/4G). Similar examples were found for an exonic SNV at scaffold_37: 17305121 or chr19:17555816 (Supplementary Figure 4C/4D) and an intronic SNV at scaffold_12:48083060 or chr10:114357477 (Supplementary Figure 4E/4F). Such discrepancies reflect the underlined genomic sequence differences between the personalized HCC1395BL_v1.0 and the common reference GRCh38, illustrating the importance of using personal genome for accurate somatic mutation discovery and subsequent analysis such as SNP genotyping assay design and validation as the mismatches in allele-specific probes or primers would impact melting temperature and binding efficiency for a genotyping assay.

Among those 43,285 somatic SNVs supported by both Strelka2 and MuTect2 on HCC1395BL_v1.0 (Supplementary Table 6), 2,790 SNVs were identified without the equivalent GRCh38-based SNVs, and from them, 1,017 sites were well-supported by more than 10 alternate allele reads with the percentage of alternate allele read coverage at least $50 \%$. By colocating these SNVs with RefSeq genes and transcripts mapped on HCC1395BL_v1.0, 522 of 2,790 SNVs were found in exonic or intronic regions, while 177 SNVs of 1,017 SNVs were overlapping with 71 gene regions (exonic or intronic), suggesting that some meaningful somatic SNVs could be overlooked when using GRCh38 as the reference genome (Figure 4D).

With respect to structural variation identification, more balanced counts of large insertions and deletions were observed on HCC1395BL_v1.0 as reference when alignment-based SV calling tools, such as pbsv (Figure 5A/5B) and Sniffles (Figure 5C/5D) were used. However, as previously reported $[14,26]$, more insertion calls were identified than deletions on the GRCh38 reference. Such excessive insertion calls were likely related to deletion bias in GRCh38 reference $[14,26]$.

Previously, without a personal genome available, a common reference, such as GRCh38, was the only resource available for assembly-to-assembly based (indirect) structural variation 
discovery [27]. With an established personal genome based on the normal sample from the same individual, we were able to make a direct comparison of the assembly of the HCC1395 cancer cell line with HCC1395BL_v1.0. We continuously observed more balanced numbers of structural variations (large insertions and deletions), especially for the SVs within the ranges between 50 to 500 bps in this analysis. An Alu repeat peak ( 300 bps) and an $L 1$ repeat peak ( 6,000 bps) were seen for both insertion and deletion calls (Figure 5E/5F). By merging two alignment-based callsets with two assembly-based callsets and requiring at least two methods supporting each SV site, we identified 3,498 large deletions, 2,239 large insertions and 101 Duplications (DUP) as somatic SVs from HCC1395 cancer cell line.

A

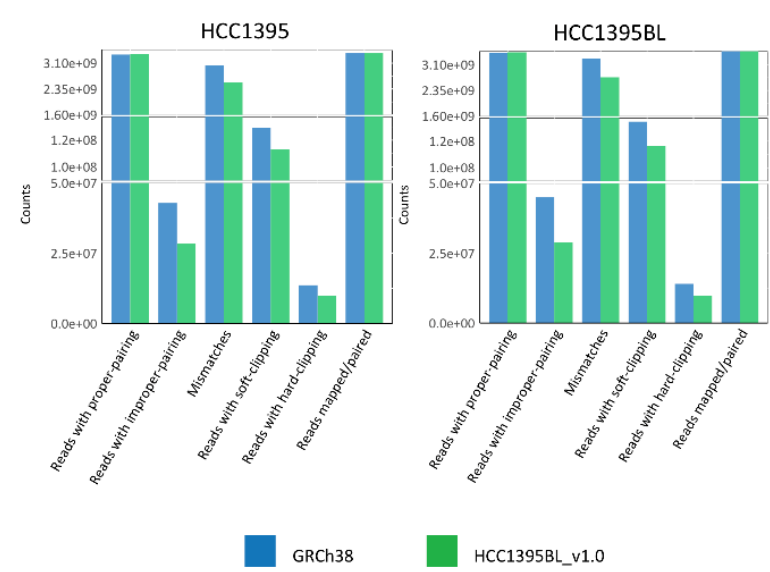

C

GRCh38 based SNVs mapping to HCC1395BL_v1.0 based SNVs

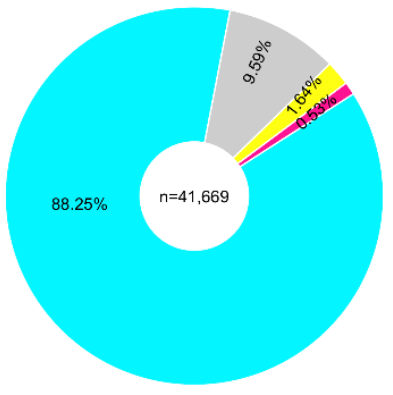

$\square$ Identical SNVs $\square$ Equivalent SNVs $\square$ Mapped but no SNVs $\square$ Not mapped

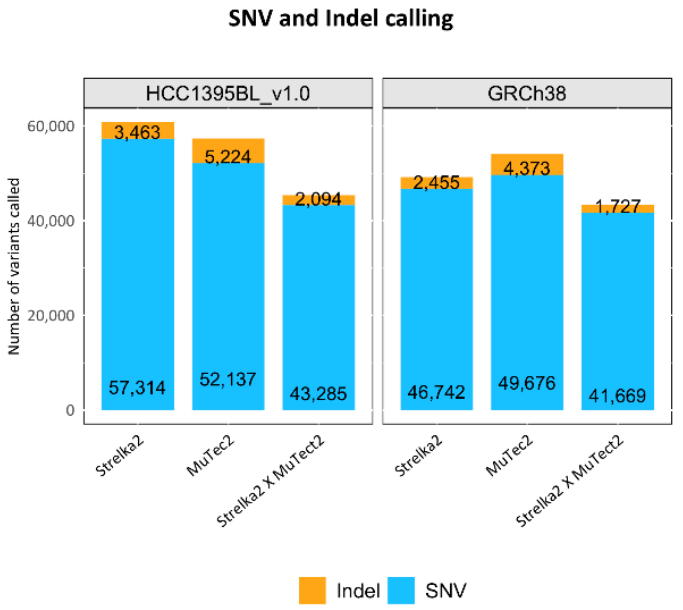

D

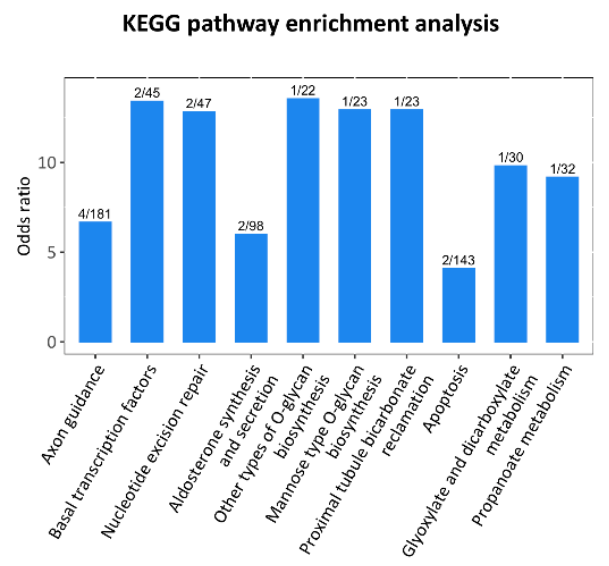

Figure 4. (A) The mappability of short reads on GRCh38 and HCC1395BL_v1.0 with gapped yaxis for visualizing the low range. The numbers of improper-paired reads, reads with mismatches, soft-clipping and hard-clipping were reduced substantially on HCC1395BL_v1.0 for 
both cell lines. (B) The sensitivity of somatic SNVs and indels detection on GRCh38 and HCC1395BL_v1.0. The numbers of overlapping variants between MuTect2 and Strelka2 were higher on HCC1395BL_v1.0. (C) 41,669 GRCh38 based somatic SNVs mapped onto HCC1395BL_v1.0 based SNVs with 40,768 (97.83\%) SNVs as identical (36,773 SNVs, $88.25 \%)$ or equivalent (3,995 SNVs, 9.59\%). 682 SNVs (1.64\%) were able to map on HCC1395BL_v1.0 but without overlapping Strelka2/MuTect2 calls. 219 SNVs (0.53\%) were considered as "notmapped" on HCC1395BL_v1.0 due to the stringent mapping criteria. (D) KEGG pathway enrichment analysis of 71 genes overlapped with the 1,017 novel SNVs detected on HCC1395BL_v1.0 as a reference. Shown here are the top 10 enriched pathways with "Odd Ratio" (zScore) on y-axis. The numeric labels are the enriched gene counts verse the total genes in each pathway.

A

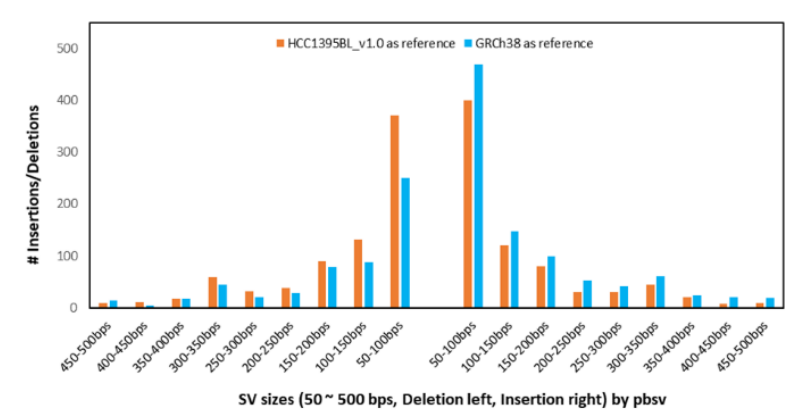

c

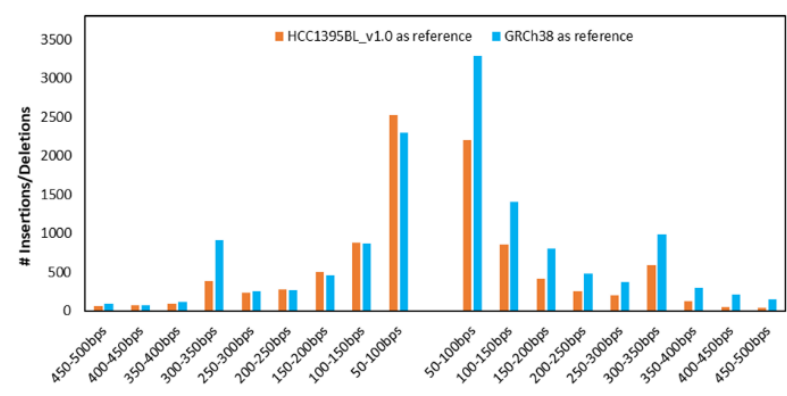

SV sizes ( $50 \sim 500$ bps, Deletion left, Insertion right) by Sniffles

E

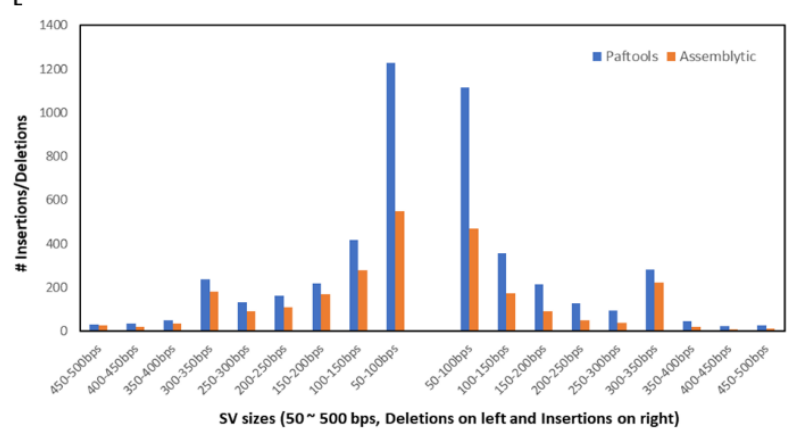

B

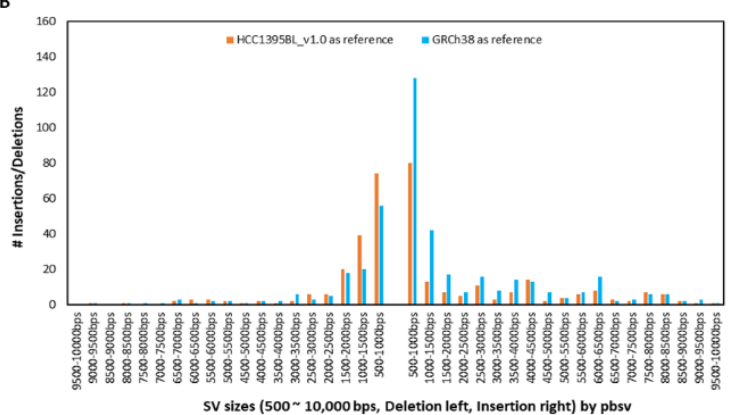

D
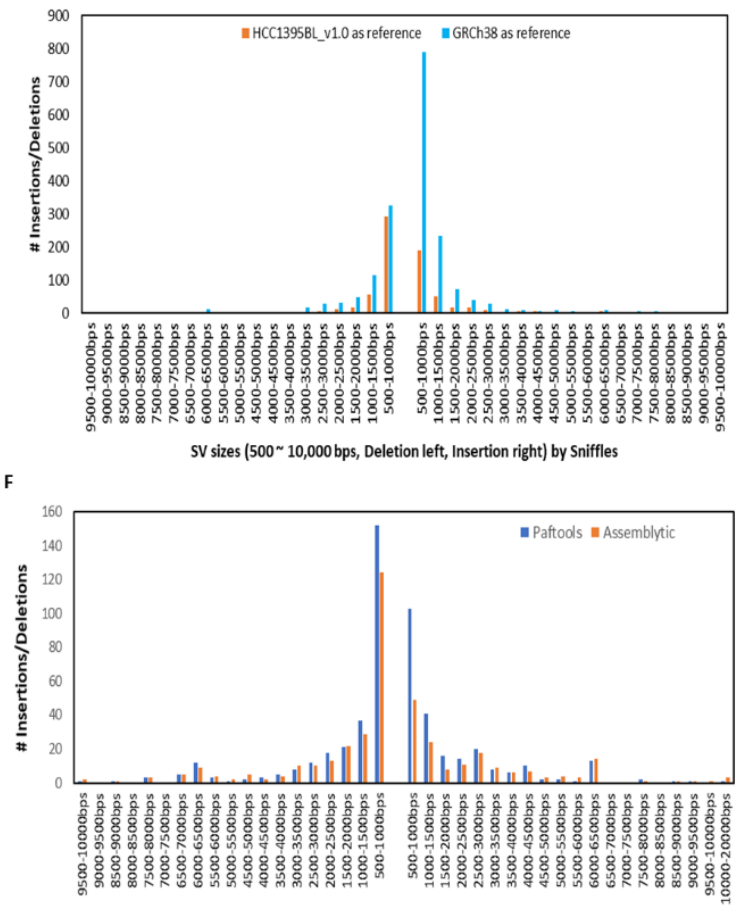

SV sizes (500 10,000 bps, Deletions on left and Insertions on right) 
Figure 5. Size distributions of insertions and deletions called by pbmm2/pbsv and Sniffles using HCC1395BL_v1.0 and GRCh38 as references, respectively: (A) $50-500$ bps by pbsv; (B) $500-$ 10,000 bps by pbsv; (C) $50-500$ bps by Sniffles; (D) $500-10,000$ bps by Sniffles. Size distribution of SVs detected from direct assembly comparison of HCC1395 to HCC1395BL using paftools and Assemblytics: (E) $50-500$ bps; (F) $500-10,000$ bps.

\section{Complete mitochondrial genome assemblies reveal non-synonymous somatic mutations}

Mitochondria are considered the powerhouse of the cell. However, mitochondrial genome assemblies in recent published de novo assemblies have either been absent or highly fragmented [28]. In this study, we completely assembled the mitochondrial genomes for both HCC1395BL and HCC1395 cell lines into single contigs. By directly comparing the two mitochondrial genomes, we identified two undocumented non-synonymous somatic mutations (T4813C and C4938A, not collected in dbSNP153 release) in the MT-ND2 gene, and one nonsynonymous somatic mutation (G14249A) on MT-ND6 gene (Figure 6). We manually checked those 3 mutations on the visualization tool IGV and confirmed that those 3 mutations have strong evidence supports. To our knowledge, those 3 somatic mutations were not reported previously in breast cancer [29-32]. Both MT-ND2 and MT-ND6 genes are subunits of the respiratory chain Complex I in the mitochondrial inner membrane, which often contains tumorigenesis related variants $[33,34]$; therefore these amino-acid changing alterations may be detrimental to the normal function of the cellular system and should be further investigated. 
A

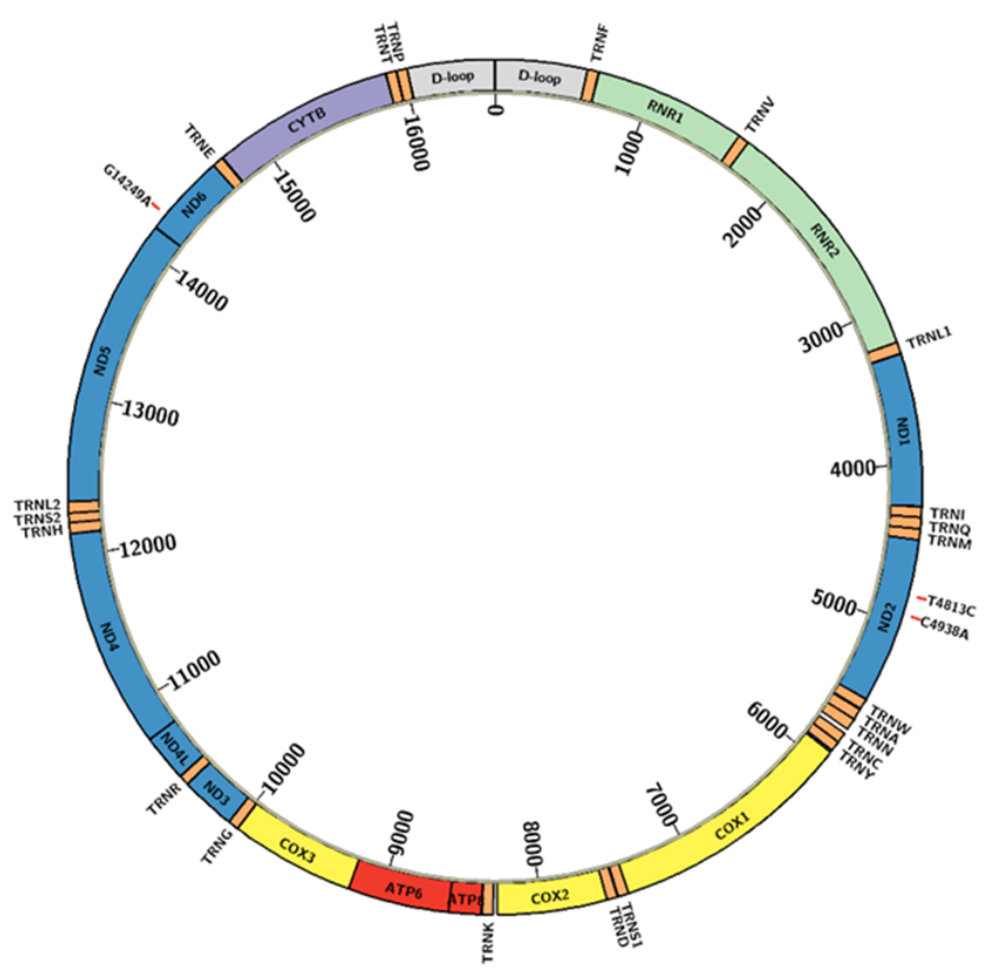

B

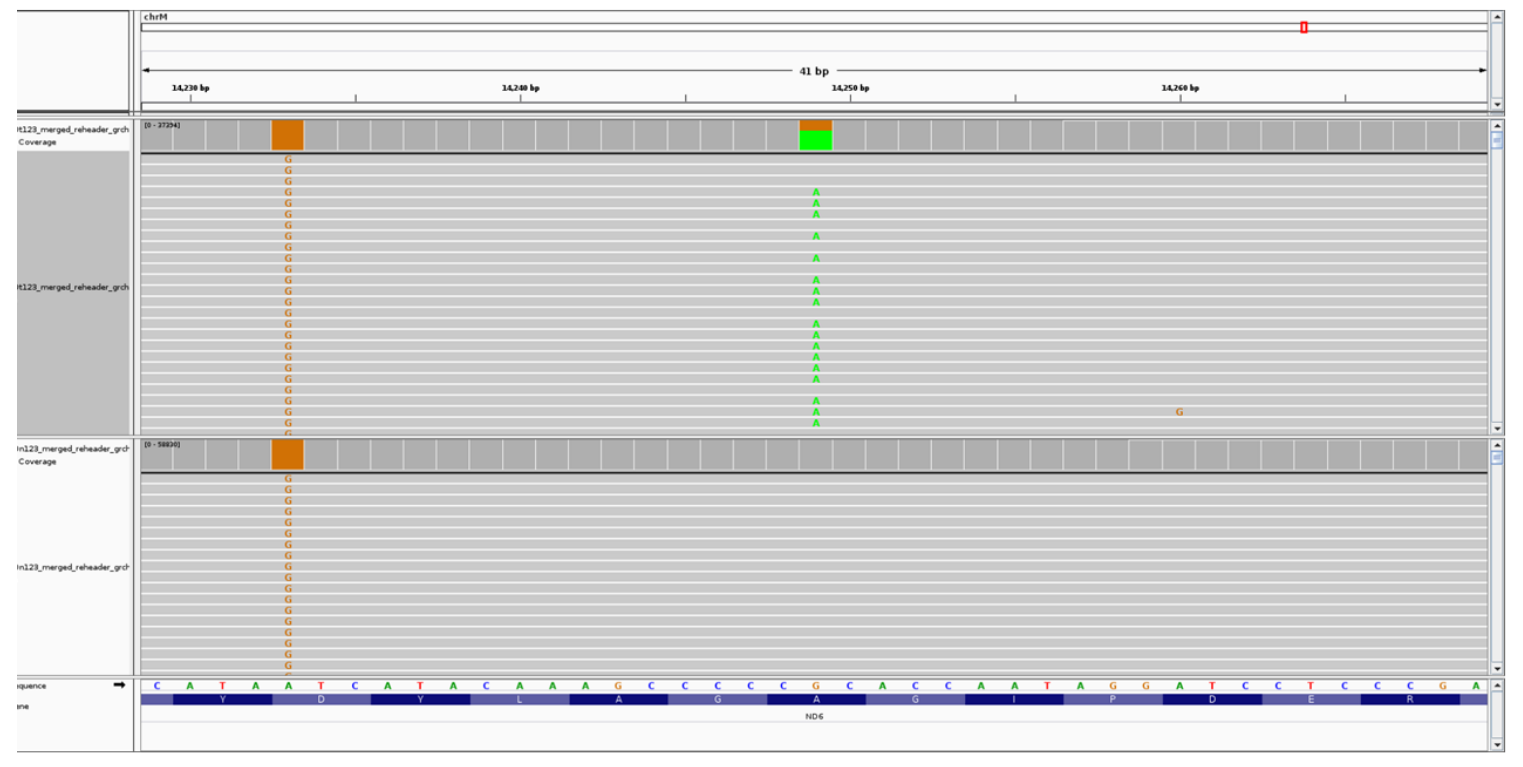

Figure 6. (A) Mitochondrial genome of HCC1395BL/HCC1395 with three unreported nonsynonymous somatic mutations (T4813C and C4938A on MT-ND2 gene, and G14249A on MTND6 gene), which were confirmed on IGV for G14249A in HCC1395 cell line only, but not in HCC1395BL cell line (B). 


\section{Discussion}

In this study, we used a combination of multiple sequencing technologies, including short reads, linked-reads, and long reads to construct the first de novo assemblies of a tumor-normal pair from the same individual with breast cancer. We subsequently applied the well-assembled genome as a reference, in comparison to using the common human reference GRCh38, for somatic variation detection and demonstrated the advantages of using a personalized genome as a reference.

Based on our analysis, high quality assembly has been achieved based on our existing data for $\mathrm{HCC} 1395 \mathrm{BL}$ in terms of contiguity and gene content. Complex genomic regions have also been well assembled, as we demonstrated that the complete HLA region was assembled into a single scaffold for HCC1395BL with an individualized haplotype. Some clinically relevant genes such as GSTT1 and KIR2DL5 (KIR2DL5A), which are not present in the chromosomes of the GRCh38 primary assembly commonly used for alignment-based analyses, were also captured in our de novo HCC1395BL assembly.

For the first time to our knowledge, we were able to identify cancer somatic mutations based on the de novo assembly from the same person with breast cancer, instead of inferring them from alignments to a common reference benchmark such as GRCh38 [10]. Our analysis showed that the de novo assembly improved read mapping, resulting in a greater percentage of properly-mapped mate pair reads, reducing total numbers of mismatches, and many fewer reads with improper-pairing, soft-clipping, and hard-clipping. Discovery of somatic SNVs and small indels was also more consistent by different calling algorithms, and large insertions/deletions were more balanced in numbers when the de novo personal assembly was used for variant calling from this paired reference samples. Mapping analysis of GRCh38-based somatic SNVs set with flankings to the de novo assembly revealed that small percentage (1.64\%) of GRCh38 based SNVs have good mapping locations on personal genome but without corresponding SNV calls, indicating potential false positives exist. Some SNVs (9.59\%) may have 
the same reference/alternate alleles on de novo assembly as on GRCh38 reference genome, but their flanking sequences may be slightly different, highlighting the critical needs of promoting the personal genome assembly for individualized medical research.

Furthermore, we also finished the first complete mitochondrial genomes for a tumor-normal pair. As a result, we were able to perform genome-wide comparative analysis of these two genomes on the personal genome level, resulting in more accurate and complete results from nuclear genome to mitochondrial genome. Our results illustrate that using the de novo genome for somatic variant discovery from tumor-normal paired data is possible. We showed that accurate detection of mutation enabling precision cancer medicine can be achieved with a personalized genome, though the overall cost of the sequencing and subsequent computational analysis is substantial.

As sequencing technology continues to evolve with longer read length and lower per-base error rate, many genomic regions such as telomeres and centromeres that were previously impossible to assemble, and whose biology is consequently poorly understood, are now within reach of being finished [14]. In fact, Human Pangenomes Consortium (https://humanpangenome.org/) has been working on constructing a more representative genome reference, with the goal of producing 300 high quality haplotype resolved human genomes from different human sub-populations. Such new developments should encourage the Consortium and other researchers to continue improving the quality of the de novo personal assembly for this tumor-normal pair by applying PacBio's HiFi reads and Oxford Nanopore's ultra-long reads in the near future. Such advancement in genome assembly will offer a better path forward for somatic variant identification using personalized genome as a reference, and thus provide more insights into understanding how tumorigenesis occurs from the molecular level, leading to discovery of vital genetic markers for cancer diagnostic and drug development. 


\section{Methods}

\section{Whole genome sequencing datasets}

A matched tumor/normal pair of TNBC breast cancer cell lines (HCC1395 as tumor and HCC1395BL as normal) was selected as reference samples for whole genome sequencing with multiple platforms. For HCC1395BL assembly analysis, included were about 175-fold of Illumina short reads, 161-fold of 10X Genomics (10X) linked reads, 53-fold of Pacific Bioscience (PacBio) long reads, 71-fold of Hi-C reads, and 15-fold Oxford Nanopore technologies (ONT) reads, respectively (Table S1). For HCC1395, about 170-fold of Illumina short reads, 160-fold of 10X Genomics linked reads, and 46-fold of Pacific Bioscience (PacBio) long reads were included. Library preparations and sequencing for Illumina short reads, 10X linked reads and PacBio long reads were described previously [46-47].

Dovetail HiC library preparation and sequencing: The Dovetail HiC libraries were prepared for each sample in a similar manner as described previously (Erez Lieberman-Aiden et al., 2009). For each library, chromatin was fixed in place with a $1 \%$ formaldehyde solution in the nucleus and then extracted. Fixed chromatin was digested with Dpnll, the 5' overhangs were filled in with biotinylated nucleotides, and then free blunt ends were ligated. After ligation, crosslinks were reversed, and the DNA purified from protein. Purified DNA containing biotinylated freeends were removed as those are not reflective of proximity-ligated molecules. The DNA was then sheared to $350 \mathrm{bp}$ mean fragment size and sequencing libraries were generated using NEBNext Ultra enzymes and Illumina-compatible adapters. Internal biotin-containing fragments were isolated using streptavidin beads before PCR enrichment of each library. The libraries were sequenced on an Illumina HiSeq $X$ to a depth of $200 \mathrm{M}$ read pairs per library.

Oxford Nanopore technologies (ONT) MinION sequencing data: Genomic DNA from HCC1395BL cell line was extracted using QIAGEN MagAttract HMW DNA Kit (QIAGEN, Hilden, Germany). 1ug of initial DNA without fragmentation was used for library construction using SQK-LSK109 ligation sequencing kit (Oxford Nanopore Technologies, Oxford, UK). Library preparations were 
conducted as per the protocols provided by ONT. Each library was sequenced on an individual MinION FLO-MIN106D R9.4 flowcell. Prior to sequencing, flowcell pore counts were measured using the MinKNOW Platform QC script (Oxford Nanopore Technologies, Oxford, UK). About 300ng of completed libraries were loaded as per instructions from ONT. Raw sequence reads were basecalled in real time via MinKNOW. Basecalled data passing quality parameters (qmean > 7) were converted to fastq. Only reads designated as pass were included in further analyses.

\section{Assembly, polishing, Scaffolding, and Phasing}

PacBio long reads data was firstly error-corrected and then assembled into primary contigs using "canu" assembler (version 1.8) [17]. The contig sequences were then polished with Illumina paired-end reads using PILON (version 1.22) [35]. The polishing process was performed twice to achieve the best results. Linked reads from 10X Genomics were assembled using "Supernova" assembler (version 2.0.0) [18]. Scaffolding with linked-reads was performed using ARCS (version 1.0.5) [36], while scaffolding with Hi-C data was completed using SALSA (https://github.com/marbl/SALSA) [37].

After scaffolding with ARCS and SALSA, we mapped the unitig sequences, which were produced with Illumina short reads using fermikit (version r188) [38], to the scaffold assembly using BWA [39], and then used bcftools (version 1.6, https://samtools.github.io/bcftools/bcftools.html) to generate the final consensus assembly.

The Illumina short reads were aligned onto the HCC1395BL_v1.0 using BWA mem [39], and duplicated reads were marked with Picard MarkDuplicates. High confident heterozygous sites were identified using GATK4 (version gatk-4.0.3.0) [40]. Phasing was performed with the identified high confident heterozygous sites and long reads from PacBio and ONT using phasing tools WhatsHap (version 0.18) [41]. Two haplotypes of the assembly in FASTA format were also reconstructed with the phasing information. Assembly-based germline SNVs in autosomal chromosomes were called using dipcall (https://github.com/lh3/dipcall) with two haplotypes as 
inputs.

\section{Assembly evaluation}

QUAST (version 5.0.0) [42], Benchmarking Universal Single-Copy Orthologue (BUSCO, version 3.0.0) [43] were used to assess the quality of each de novo assembly. BLAT (v36) was used for mapping all RefSeq mRNA transcripts (accession prefixed with NM_and NR_) (ftp://ftp.ncbi.nlm.nih.gov/genomes/all/GCF/000/001/405/GCF 000001405.38 GRCh38.p12/G CF 000001405.38 GRCh38.p12 rna.fna.gz) that were previously annotated on the GRCh38 assembly to the new assembly with parameter minldentity 92.

For GRCh38 consistency analysis, each assembly was compared with GRCh38 reference assembly ( $\underline{\mathrm{ftp}: / / \mathrm{ftp}-}$

trace.ncbi.nlm.nih.gov/ReferenceSamples/seqc/technical/reference genome/ GRCh38/GRCh38.d1.vd1.fa) using minmap2 [44]. Alignments with mapping quality 60 and alignment length $100 \mathrm{~Kb}+$ were considered as good links for the consistency plot by Circos (Krzywinski, M., et al., 2009). Scaffolds smaller than $10 \mathrm{~kb}$ were excluded from the analysis.

We also introduced a new parameter "Top50", which is the summed length of the 50 longest scaffolds, to monitor the contiguity of a given assembly during scaffolding process, as the longread and $\mathrm{Hi}-\mathrm{C}$ sequencing technologies could make it possible to have arm-scale or chromosomal scale assembly. For the human genome with a total of 48 chromosomal arms, Top50 might be a suitable indicator to reflect the contiguity of the scaffold assembly if each chromosomal arm forms a scaffold.

\section{Genome annotation}

To better annotate the final assembly HCC1395BL_v1.0, BLAT (version 36) and AUGUSTUS (version 3.3.1) pipeline [45] was used to map the previously described RefSeq transcripts to the assembly (excluding all pseudogenes and genes from NC_000024 chromosome Y). Proteincoding transcripts with annotations containing "pseudogene" and non-protein-coding 
transcripts with annotations containing "pseudo=true" in their deflines were considered as "pseudogenes" in this analysis. For BLAT, the option "minldentity" was set to 92. Transcripts with more than 95\% alignment coverage and 95\% ungapped identity were considered mapped on HCC1395BL_v1.0 assembly. One exception was applied to HLA-DQA1 (NM_002122.3) gene that was $100 \%$ covered by the HCC1395BL_v1.0, but with the identity of $92.95 \%$, for comparing the HLA haplotypes between GRCh38 and HCC1395BL_v1.0. No other mapped location was found for HLA-DQA1 gene on HCC1395BL_v1.0. In case of multiple mapping locations, the best mapping location with maximum number of matching bases for the transcript was selected.

\section{Somatic variant detection}

BWA [39] was used to align the Illumina short reads onto the de novo assembly (HCC1395BL_v1.0) and GRCh38 primary assembly, respectively. Duplicate reads were marked with Picard MarkDuplicates. Mapping statistics was gathered using samtools with the "stats" option (http://www.htslib.org/doc/samtools-stats.html).

Strelka2 (version 2.9.2) [23] and MuTect2 (version gatk-4.0.3.0/gatk Mutect2) [24] were used to generate somatic SNVs and indels. The chrX, chr6p and chr16q regions were not included for somatic variant comparison, for consistency with the reference somatic set (v1.1) from the SEQC2 Somatic Mutation Working Group [46-47].

Structural variations from PacBio long reads data were identified using pbmm2/pbsv (version 2.2.1, https://github.com/PacificBiosciences/pbsv) and Sniffles (version 1.0.11, https://github.com/fritzsedlazeck/Sniffles). For each reference assembly, SVs were called separately using PacBio long read alignments of HCC1395 and HCC1395BL, then the SV calls of HCC1395 were filtered with SVs of HCC1395BL. Sniffles callsets were filtered with AF >= 0.9 and $10 \sim 100$ read supports.

Assembly-based SVs were generated from direct assembly comparisons of HCC1395 cancer cell line contig assembly with the de novo HCC1395BL assembly using paftools [44] and 
Assemblytics (https://github.com/MariaNattestad/assemblytics). For consistency with SEQC2 Somatic Mutation Working Group [46-47], calls from chrX, chr6p, and chr16q were also excluded for comparison. SURVIVOR (version 1.0.7, https://github.com/fritzsedlazeck/SURVIVOR) was used for merging and filtering SV callsets.

\section{Mapping GRCh38 SNVs to de novo assembly}

Two-steps mapping approaches were performed to find corresponding locations of the GRCh38-based somatic SNVs on the de novo assembly. We extracted both the reference and alternate alleles of each SNV with their 50bps flanking sequences from GRCh38 and created a fasta file before mapping using BLAST (blast 2.10.1). The first step was to map all SNVs with higher criteria so that SNVs with identity >=99\% and alignment length >=101 bps were selected. The un-selected SNVs from Step1 were then mapped in the second step with lower thresholds (using 95\% identity and 95 bps alignment coverage as cut-off based on our mapping experiments) so that best mapped SNVs with some mismatches and small indels would be selected (Figure S6). For both steps, both alleles of each SNV were required to map onto the same locations with identical start and end on de novo assembly. In addition, to be considered as an equivalent SNV call between GRCh38 and de novo assembly, the alternate allele must be at the center position. Manual inspections on IGV for some SNVs were also performed. Unselected SNVs from Step2 were considered as unmapped.

\section{Variant annotation and pathway analysis}

Variant function analysis was performed using ANNOVAR [25]. Pathway analysis were performed through Enrichr web site (https://maayanlab.cloud/Enrichr/).

\section{Mitochondrial sequence analysis}

Contigs from HCC1395BL assembly and HCC1395 assembly that were fully covering the mitochondrial sequences from GRCh38 (16,569 bps; https://www.ncbi.nlm.nih.gov/nuccore/NC 012920.1) were selected based on minimap2 mapping results. Since the mitochondrial genome is circular, the full mitochondrial sequences 
were extracted from each of the selected contigs based on BLAST mapping results. CLUSTAL (v1.2.4) was used to generate multiple sequence alignments for variant analysis. The variants were annotated with the MITOMAP human mitochondrial genome database (http://www.mitomap.org, 2019) and dbSNP (v153).

\section{Data availability}

All raw data are available in NCBI SRA database (SRP162370). The HCC1395BL_v1.0 assembly has been deposited at DDBJ/ENA/GenBank under the accession JAEOAY000000000. Final de novo assembly fasta file and de novo assembly-based somatic mutation VCF files are also accessible via the NCBI ftp site ( trace.ncbi.nlm.nih.gov/ReferenceSamples/seqc/Somatic Mutation WG/assembly).

\section{Code and software availability}

All software or tools used for de novo genome assemblies, assembly evaluations, and variant calls were publically available and listed in "Methods" section.

\section{References}

1. Berger, M.F. and E.R. Mardis, The emerging clinical relevance of genomics in cancer medicine. Nat Rev Clin Oncol, 2018. 15(6): p. 353-365.

2. Malone, E.R., et al., Molecular profiling for precision cancer therapies. Genome Med, 2020. 12(1): p. 8.

3. Cancer Genome Atlas, N., Comprehensive molecular portraits of human breast tumours. Nature, 2012. 490(7418): p. 61-70.

4. Consortium, I.T.P.-C.A.o.W.G., Pan-cancer analysis of whole genomes. Nature, 2020. 578(7793): p. 82-93.

5. Rosenfeld, J.A., C.E. Mason, and T.M. Smith, Limitations of the human reference genome for personalized genomics. PLoS One, 2012. 7(7): p. e40294.

6. Schneider, V.A., et al., Evaluation of GRCh38 and de novo haploid genome assemblies demonstrates the enduring quality of the reference assembly. Genome Res, 2017. 27(5): p. 849-864.

7. Xiao, W., et al., Challenges, Solutions, and Quality Metrics of Personal Genome Assembly in Advancing Precision Medicine. Pharmaceutics, 2016. 8(2).

8. Yang, X., et al., One reference genome is not enough. Genome Biol, 2019. 20(1): p. 104. 
9. Ballouz, S., A. Dobin, and J.A. Gillis, Is it time to change the reference genome? Genome Biol, 2019. 20(1): p. 159.

10. Eichler, E.E., Genetic Variation, Comparative Genomics, and the Diagnosis of Disease. N Engl J Med, 2019. 381(1): p. 64-74.

11. Shi, L., et al., Long-read sequencing and de novo assembly of a Chinese genome. Nat Commun, 2016. 7: p. 12065.

12. Seo, J.S., et al., De novo assembly and phasing of a Korean human genome. Nature, 2016. 538(7624): p. 243-247.

13. Jain, M., et al., Nanopore sequencing and assembly of a human genome with ultra-long reads. Nat Biotechnol, 2018. 36(4): p. 338-345.

14. Miga, K.H., et al., Telomere-to-telomere assembly of a complete human X chromosome. Nature, 2020.

15. Shumate, A., et al., Assembly and annotation of an Ashkenazi human reference genome. Genome Biol, 2020. 21(1): p. 129.

16. Gazdar, A.F., et al., Characterization of paired tumor and non-tumor cell lines established from patients with breast cancer. Int J Cancer, 1998. 78(6): p. 766-74.

17. Koren, S., et al., Canu: scalable and accurate long-read assembly via adaptive k-mer weighting and repeat separation. Genome Res, 2017. 27(5): p. 722-736.

18. Weisenfeld, N.I., et al., Direct determination of diploid genome sequences. Genome Res, 2017. 27(5): p. 757-767.

19. Stephens, P.J., et al., Complex landscapes of somatic rearrangement in human breast cancer genomes. Nature, 2009. 462(7276): p. 1005-10.

20. Chin, C.S., et al., A diploid assembly-based benchmark for variants in the major histocompatibility complex. Nat Commun, 2020. 11(1): p. 4794.

21. Satta, Y., W.E. Mayer, and J. Klein, HLA-DRB intron 1 sequences: implications for the evolution of HLA-DRB genes and haplotypes. Hum Immunol, 1996. 51(1): p. 1-12.

22. Cai, L., et al., In-depth comparison of somatic point mutation callers based on different tumor next-generation sequencing depth data. Sci Rep, 2016. 6: p. 36540.

23. Kim, S., et al., Strelka2: fast and accurate calling of germline and somatic variants. Nat Methods, 2018. 15(8): p. 591-594.

24. Cibulskis, K., et al., Sensitive detection of somatic point mutations in impure and heterogeneous cancer samples. Nat Biotechnol, 2013. 31(3): p. 213-9.

25. Wang, K., M. Li, and H. Hakonarson, ANNOVAR: functional annotation of genetic variants from high-throughput sequencing data. Nucleic Acids Res, 2010. 38(16): p. e164.

26. Chaisson, M.J., et al., Resolving the complexity of the human genome using singlemolecule sequencing. Nature, 2015. 517(7536): p. 608-11.

27. Nattestad, M., et al., Complex rearrangements and oncogene amplifications revealed by long-read DNA and RNA sequencing of a breast cancer cell line. Genome Res, 2018. 28(8): p. 1126-1135.

28. Mai, Z., et al., Misassembly of long reads undermines de novo-assembled ethnicityspecific genomes: validation in a Chinese Han population. Hum Genet, 2019. 138(7): p. 757-769.

29. Zhu, W., et al., Mitochondrial DNA mutations in breast cancer tissue and in matched 
nipple aspirate fluid. Carcinogenesis, 2005. 26(1): p. 145-52.

30. Tseng, L.M., et al., Somatic mutations of the mitochondrial genome in human breast cancers. Genes Chromosomes Cancer, 2011. 50(10): p. 800-11.

31. Jimenez-Morales, S., et al., Overview of mitochondrial germline variants and mutations in human disease: Focus on breast cancer (Review). Int J Oncol, 2018. 53(3): p. 923-936.

32. Ban, M., et al., Investigation of the role of mitochondrial DNA in multiple sclerosis susceptibility. PLoS One, 2008. 3(8): p. e2891.

33. Brandon, M., P. Baldi, and D.C. Wallace, Mitochondrial mutations in cancer. Oncogene, 2006. 25(34): p. 4647-62.

34. Hertweck, K.L. and S. Dasgupta, The Landscape of mtDNA Modifications in Cancer: A Tale of Two Cities. Front Oncol, 2017. 7: p. 262.

35. Walker, B.J., et al., Pilon: an integrated tool for comprehensive microbial variant detection and genome assembly improvement. PLoS One, 2014. 9(11): p. e112963.

36. Yeo, S., et al., ARCS: scaffolding genome drafts with linked reads. Bioinformatics, 2018. 34(5): p. 725-731.

37. Ghurye, J., et al., Scaffolding of long read assemblies using long range contact information. BMC Genomics, 2017. 18(1): p. 527.

38. Li, H., Fermikit: assembly-based variant calling for Illumina resequencing data. Bioinformatics, 2015. 31(22): p. 3694-6.

39. Li, H. and R. Durbin, Fast and accurate short read alignment with Burrows-Wheeler transform. Bioinformatics, 2009. 25(14): p. 1754-60.

40. DePristo, M.A., et al., A framework for variation discovery and genotyping using nextgeneration DNA sequencing data. Nature Genetics, 2011. 43(5): p. 491-+.

41. Patterson, M., et al., WhatsHap: Weighted Haplotype Assembly for Future-Generation Sequencing Reads. J Comput Biol, 2015. 22(6): p. 498-509.

42. Gurevich, A., et al., QUAST: quality assessment tool for genome assemblies. Bioinformatics, 2013. 29(8): p. 1072-5.

43. Waterhouse, R.M., et al., BUSCO applications from quality assessments to gene prediction and phylogenomics. Mol Biol Evol, 2017.

44. Li, H., Minimap2: pairwise alignment for nucleotide sequences. Bioinformatics, 2018. 34(18): p. 3094-3100.

45. Stanke, M., et al., Using native and syntenically mapped cDNA alignments to improve de novo gene finding. Bioinformatics, 2008. 24(5): p. 637-44.

46. Li Tai Fang, et al., Establishing community reference samples, data and call sets for benchmarking cancer mutation detection using whole-genome sequencing. Nat. Biotechnol. (in the press).

47. Wenming Xiao, et al., Towards best practice in cancer mutation detection with wholegenome and whole-exome sequencing. Nat. Biotechnol. (in the press). 


\section{Acknowledgements}

The genomic work carried out at the LLU Center for Genomics was funded in part by the National Institutes of Health (NIH) grant S100D019960 (CW). The study was also partially supported by the American Heart Association grant 18IPA34170301 (CW). Chunlin Xiao and Valerie Schneider were supported by the Intramural Research Program of the National Library of Medicine, National Institutes of Health. Javkhlan Ganbat provided useful help regarding HiC data analysis.

\section{Author contributions}

CX, WX designed the study and wrote the manuscript draft. CP performed HiC sequencing and QA/QC. ZC, WC, TL and CW performed ONT sequencing and QA/QC. CX, WX, WC, ZC, CW, and LF performed the analyses. CX, WX, WC, ZC, CW, CP, and VS improved the manuscript. CX, VS and WX managed the data.

\section{Competing interests}

CP was employed by Dovetail Genomics, LLC, and LF was employed by Roche Sequencing Solutions Inc during the course of this research. The other authors declare no competing interests.

\section{Disclaimer}

The content of this manuscript is solely the responsibility of the authors, and the views presented here do not necessarily reflect official policy of the US Food and Drug Administration or US National Institutes of Health. Any mention of commercial products or materials or tools is purely for clarification purpose and not intended as endorsement or discouragement. 